\title{
THE EVOLUTION OF AGB STARS IN THE MAGELLANIC CLOUDS
}

\author{
B.E. WESTERLUND ${ }^{1}$, M. AZZOPARDI ${ }^{2}$, J. BREYSACHER ${ }^{3}$, E. REBEIROT ${ }^{2}$ \\ 1 Astronomical Observatory, Uppsala University, Box 515, S-751 20 Uppsala, Sweden \\ 2 Observatoire de Marseille, 2 Place Le Verrier, F-13248 Marseille, Cedex 4, France \\ 3 European Southern Observatory, Karl-Schwarzschild-Str. 2, D-8046 Garching bei \\ München, Germany
}

ABSTRACT. Photoelectric observations of over $100 \mathrm{C}$ stars in the Small Magellanic Cloud (SMC) and 20 in the Large Magellanic Cloud (LMC) were carried out with the Johnson JHK system using the standard ESO equipment on the $3.6 \mathrm{~m}$ telescope. The limiting magnitude is about $\mathrm{K}=13.7$.

\section{Introduction}

We have used the GRISM spectra (spectral range 4350 - 5300 $\AA$; dispersion $2200 \AA / \mathrm{mm}$ ) to select stars for spectroscopy and JHK photometry with a good coverage in magnitude, colour and carbon content. Spectra of about 50 stars in the SMC and 15 in the LMC have been obtained with the Boller and Chivens spectrographs on the ESO 3.6 and 2.2m telescopes and with the ESO Faint Object Spectrograph and Camera (EFOSC), mainly with CCD chips as detectors. The dispersions are between 114 and $230 \AA / \mathrm{mm}$ and the spectral range covered is $4500 \AA$ to $7000 \AA$. The faintest star observed has $K=13.4 \mathrm{mag}$. The spectra have been analyzed with the aid of the ESO IHAP system.

\section{Measurements}

We have measured the $\mathrm{C}_{2}$ and $\mathrm{CN}$ strengths in the spectra as equivalent widths between 4820 and $5220 \AA$ and 5720 and $5840 \AA$, respectively; the integrated intensity of the D-lines; a gradient using the "windows" between 6770 and $4820 \AA$; and the ${ }^{12} \mathrm{CN}$ and ${ }^{13} \mathrm{CN}$-band strengths at 5750 and $5790 \AA$. The quantities are used to separate $\mathrm{C}_{2}$-poor and $\mathrm{C}_{2}$-rich stars and to identify J-type stars (Bouigue 1954) and C-stars with low $\mathrm{CN}$ content. A comparison of the present ${ }^{12} \mathrm{CN} /{ }^{13} \mathrm{CN}$ ratios with Gow's (1977, Fig. 7) shows that our J-type stars have an isotope ratio of ${ }^{12} \mathrm{C} /{ }^{13} \mathrm{C} \approx 4$; the normal and $\mathrm{C}_{2}$-poor stars have ${ }^{12} \mathrm{C} /{ }^{13} \mathrm{C} \approx 20-25$.

We have used published JHK photometry in addition to our own photometry. All data have been transferred to the Johnson JHK system (cf. Frogel et al. 1978). Reddening corrections are from Cohen et al. (1981) or for clusters as suggested by the various observers. To facilitate comparisons with previous work we have used $\mathrm{m}-\mathrm{M}=18.6$ for the $\mathrm{LMC}$ and $19.1 \mathrm{mag}$ for the SMC. Bolometric absolute magnitudes, $\mathbf{M}_{\text {bol }}$, and effective temperatures, $\log \mathbf{T}_{\text {eff, }}$ have been calculated following Wood et al. (1983) and Bessell et al. (1983), respectively. 


\section{The Hertzsprung-Russell diagrams}

We have identified the early Asymtotic Giant Branches (AGB) for various age groups using published JHK photometry of M and S stars in clusters of known SWB (Searle et al. 1980) classes and identified transition regions. The field carbon stars in the Magellanic Clouds follow evolutionary sequences from the $\mathrm{M}-(\mathrm{S})$ - $\mathrm{C}$ transition regions beginning as $\mathrm{C}_{2}$-poor stars. For the least massive stars (SWB class VII) the transition occurs directly from $\mathrm{M}$ to $\mathrm{C}$. Some of them pass through a J-type stage. The $\mathrm{C}_{2}$ strength increases as the star approaches the final stage of Miratype.

The most massive $\mathrm{C}$ stars are in the $\mathrm{LMC}$ and are often of J-type, and $\mathrm{CO}$ rich. They may be related to the most luminous of the two M-giant branches (Frogel \& Blanco 1983). If their masses are between 3.3 and $5 \mathrm{M}_{\mathrm{o}}$ they may have converted ${ }^{12} \mathrm{C}$ to ${ }^{13} \mathrm{C}$ in a hot-bottom burning process (Renzini \& Voli 1981). With a falling temperature the dredged-up ${ }^{12} \mathrm{C}$ will again dominate. Eventually, a conversion of ${ }^{12} \mathrm{C}$ to ${ }^{14} \mathrm{~N}$ occurs and luminous MS stars may form. The most luminous AGB star in the SMC, the MS variable HV11452, is more luminous than all the LMC stars in our sample. Its predecessor may have been over $5 \mathrm{M}_{\mathrm{o}}$ and evolved without passing the Cstar stage. C stars of average mass end as luminous Ce stars of nearly the same luminosity as the MS stars but much redder.

The carbon stars in the SMC cover a range in $\mathrm{M}_{\text {bol }}$ from -3 mag to $-5.9 \mathrm{mag}$. In the $\mathrm{LMC}$ the range is $-3.8 \mathrm{mag}$ to $-6.5 \mathrm{mag}$. The transition from $\mathrm{M}$ via $\mathrm{S}$ to $\mathrm{C}$ occurs in both Clouds at about $\mathrm{M}_{\mathrm{bol}} \approx-4.6$ but at a $\log \mathrm{T}_{\mathrm{e}}$ about 0.05 higher in the SMC. In the SMC the early AGB branches are blue-shifted relative to those in the LMC. The $\mathrm{C}_{2}$-poor stars fall in the $\mathrm{M}$-(S)-C transition regions with J-type stars next to them. The faintest stars form a sequence extending from the top of the early AGB-branch of class VII. They represent an older and lower-mass population.

The coolest of the more luminous J-type stars in the SMC as well as in the LMC have the strongest ${ }^{13} \mathrm{C}$. Both have $\log \mathrm{T}_{\mathrm{e}}=3.47$.

\section{References}

Bessell, M.S., Wood, P.R., Lloyd Evans, T. (1983), M.N.R.A.S. 202, 59.

Bouigue, R. (1954), Ann. d'Astrophys. 17, 104.

Cohen, J.G., Frogel, J.A., Persson, S.E., Elias, J.H. (1981), Astrophys. J. 249, 481.

Frogel, J.A., Blanco, V.M. (1983), Astrophys. J. 274, L57.

Frogel, J.A., Persson, S.E., Aaronson, M., Matthews, K. (1978), Astrophys. J. 220, 75.

Gow, C.E. (1977), Publ. Astron. Soc. Pacific 89, 510.

Renzini, A., Voli, M. (1981), Astron. Astrophys. 94, 175.

Searle, L., Wilkinson, A., Bagnuolo, W.G. (1980), Astrophys. J. 239, 803.

Wood, P.R., Bessell, M.S., Fox, M.W. (1983), Astrophys. J. 272, 99. 